\title{
No Association between Depression and Risk of Hepatocellular Carcinoma in Older People in Taiwan
}

\author{
Shih-Wei Lai, ${ }^{1,2}$ Cheng-Li Lin, ${ }^{3,4}$ Kuan-Fu Liao, ${ }^{5,6}$ and Wen-Chi Chen ${ }^{5,7}$ \\ ${ }^{1}$ School of Medicine, China Medical University, Taichung 404, Taiwan \\ ${ }^{2}$ Department of Family Medicine, China Medical University Hospital, Taichung 404, Taiwan \\ ${ }^{3}$ Department of Public Health, China Medical University, Taichung 404, Taiwan \\ ${ }^{4}$ Management Office for Health Data, China Medical University Hospital, Taichung 404, Taiwan \\ ${ }^{5}$ Graduate Institute of Integrated Medicine, China Medical University, Taichung 404, Taiwan \\ ${ }^{6}$ Department of Internal Medicine, Taichung Tzu Chi General Hospital, No. 66, Sec. 1, Fongsing Road, \\ Tanzi District, Taichung 427, Taiwan \\ ${ }^{7}$ Department of Urology, China Medical University Hospital, Taichung 404, Taiwan
}

Correspondence should be addressed to Kuan-Fu Liao; kuanfuliao@yahoo.com.tw

Received 3 September 2013; Accepted 21 October 2013

Academic Editors: A. Michael and H. P. Volz

Copyright (C) 2013 Shih-Wei Lai et al. This is an open access article distributed under the Creative Commons Attribution License, which permits unrestricted use, distribution, and reproduction in any medium, provided the original work is properly cited.

\begin{abstract}
Objectives. The objective of this study was to determine whether there is a relationship between depression and risk of hepatocellular carcinoma (HCC) in older people in Taiwan. Methods. A case-control study was conducted to analyze the database from the Taiwan National Health Insurance program. We selected 1815 subjects aged 65 years or older with newly diagnosed HCC as the case group and 7260 subjects without HCC as the comparison group, from 2000 to 2010. Both groups were compared to measure the risk of HCC. Results. After controlling for confounders, the odds ratio of HCC was 0.81 in subjects with depression (95\% confidence interval $=0.59,1.11$ ), as compared with nondepressed subjects. Conclusions. We conclude that no association is detected between depression and risk of hepatocellular carcinoma in older people in Taiwan.
\end{abstract}

\section{Introduction}

Until now, controversies exist regarding the relationship between depression and subsequent cancer risk. That is, some studies showed an increased risk $[1,2]$, but others showed no increased risk $[3,4]$. Similarly, no specific evidence is available about the relationship between depression and risk of hepatocellular carcinoma (HCC) in older people. In order to explore this issue, a population-based case-control study was conducted to analyze the database from the Taiwan National Health Insurance program.

\section{Methods}

The details of the Taiwan National Health Insurance program can be documented in previous studies [5-7]. In this casecontrol study, we randomly selected 1815 subjects aged 65 years or older with newly diagnosed HCC as the case group (1132 men and 683 women, mean age 74.31 years, and standard deviation 6.29 years) (according to the International Classification of Diseases 9th Revision-Clinical Modification, ICD-9 codes 155, 155.0, and 155.2) and 7260 subjects without HCC as the comparison group (4528 men and 2732 women, mean age 74.09 years, and standard deviation 6.53 years). Both groups were matched with sex, age, and index year of diagnosing HCC, from 2000 to 2010. The index date was defined as the date of diagnosing HCC. In order to reduce the confounding effects, subjects with any cancer (ICD-9 codes 140-208), major psychiatric diseases (ICD-9 codes 291-293, 294.0, 294.8-294.9, 295, 296.0-296.1, 296.4-296.9, and 297298), other dementia (290.0-290.4, and 294.1), or mental retardation (ICD-9 codes 317-319) diagnosed before the index date were excluded. Depression (ICD-9 codes 331.0) and other comorbidities potentially associated with HCC were diagnosed before the index date [7]. 
TABLE 1: Characteristics between hepatocellular carcinoma group and comparison group.

\begin{tabular}{|c|c|c|c|c|c|}
\hline & \multicolumn{4}{|c|}{ Hepatocellular carcinoma } & \multirow{4}{*}{$P$ value } \\
\hline & \multirow{2}{*}{\multicolumn{2}{|c|}{$\begin{array}{c}\text { No } \\
N=7260\end{array}$}} & \multirow{2}{*}{\multicolumn{2}{|c|}{$\begin{array}{c}\text { Yes } \\
N=1815\end{array}$}} & \\
\hline & & & & & \\
\hline & $n$ & $(\%)$ & $n$ & $(\%)$ & \\
\hline \multicolumn{6}{|l|}{ Age group (year) } \\
\hline $65-74$ & 4260 & 58.68 & 1065 & 58.68 & 0.99 \\
\hline $75-84$ & 3000 & 41.32 & 750 & 41.32 & \\
\hline \multicolumn{6}{|l|}{ Sex } \\
\hline Men & 4528 & 62.37 & 1132 & 62.37 & 0.99 \\
\hline Women & 2732 & 37.63 & 683 & 37.63 & \\
\hline \multicolumn{6}{|c|}{ Comorbidities before index date* } \\
\hline Depression & 385 & 5.30 & 114 & 6.28 & 0.10 \\
\hline Diabetes mellitus & 1941 & 26.74 & 636 & 35.04 & $<0.0001$ \\
\hline Cirrhosis & 137 & 1.89 & 990 & 54.56 & $<0.0001$ \\
\hline Other chronic hepatitis & 1163 & 16.02 & 1033 & 56.91 & $<0.0001$ \\
\hline Hepatitis B infection & 112 & 1.54 & 361 & 19.89 & $<0.0001$ \\
\hline Hepatitis C infection & 135 & 1.86 & 600 & 33.06 & $<0.0001$ \\
\hline Alcoholism & 57 & 0.79 & 24 & 1.32 & 0.03 \\
\hline
\end{tabular}

Data are presented as the number of subjects in each group, with percentages given in parentheses.

Chi-square test comparing subjects with and without hepatocellular carcinoma.

${ }^{*}$ Comorbidities included before index date were as follows: depression (ICD-9 codes 296.2-296.3, 300.4, and 311), diabetes mellitus (ICD-9 codes 250 ), cirrhosis (ICD-9 codes 571.2, and 571.5-571.6), other chronic hepatitis (ICD-9 codes 571.40-571.41, 571.49, and 571.8-571.9), hepatitis B infection (ICD-9 codes V02.61, 070.20, 070.22, 070.30, and 070.32), hepatitis C infection (ICD-9 codes V02.62, 070.41, 070.44, 070.51, and 070.54), and alcoholism (ICD-9 codes 303, 305.00, 305.01, 305.02, 305.03, and V11.3).

TABLE 2: Odds ratio and 95\% confidence interval of hepatocellular carcinoma associated with depression and other comorbidities.

\begin{tabular}{|c|c|c|c|c|}
\hline \multirow{2}{*}{ Variable } & \multicolumn{2}{|c|}{ Crude } & \multicolumn{2}{|c|}{ Adjusted $^{\dagger}$} \\
\hline & OR & $(95 \% \mathrm{CI})$ & OR & $(95 \% \mathrm{CI})$ \\
\hline Age (per one year) & 1.01 & $(1.00,1.01)$ & 1.04 & $(1.03,1.05)$ \\
\hline \multicolumn{5}{|c|}{ Comorbidities before index date (yes versus no) } \\
\hline Depression & 1.20 & $(0.97,1.49)$ & 0.81 & $(0.59,1.11)$ \\
\hline Diabetes mellitus & 1.48 & $(1.33,1.65)$ & 1.04 & $(0.89,1.22)$ \\
\hline Cirrhosis & 62.39 & $(51.46,75.65)$ & 28.55 & $(23.17,35.19)$ \\
\hline Other chronic hepatitis & 6.93 & $(6.19,7.75)$ & 2.23 & $(1.89,2.63)$ \\
\hline Hepatitis B infection & 15.84 & $(12.72,19.73)$ & 5.87 & $(4.40,7.84)$ \\
\hline Hepatitis C infection & 26.06 & $(21.42,31.72)$ & 7.72 & $(6.00,9.93)$ \\
\hline Alcoholism & 1.70 & $(1.05,2.74)$ & 0.85 & $(0.43,1.68)$ \\
\hline
\end{tabular}

${ }^{\dagger}$ Adjusted for age, diabetes mellitus, cirrhosis, other chronic hepatitis, hepatitis B infection, hepatitis C infection, and alcoholism.

\section{Results}

We compared the basic characteristics and co-morbidities between the HCC group and the comparison group. Table 1 shows that the HCC group was more likely to have diabetes mellitus, cirrhosis, other chronic hepatitis, hepatitis B infection, hepatitis $\mathrm{C}$ infection, and alcoholism, with statistical significance. There were 114 subjects with depression among the HCC group (6.28\%) and 385 subjects with depression among the comparison group (5.30\%), without significant difference $(P=0.10)$. After controlling for confounding factors, multivariable logistic regression analysis demonstrated that the odds ratio (OR) of HCC was 0.81 in subjects with depression (95\% confidence interval $(\mathrm{CI})=0.59,1.11)$, as compared with nondepressed subjects (Table 2).

\section{Discussion}

To date, there is no consensus about the association between depression and subsequent cancer risk [1-4]. One cohort study by Chen and Lin in Taiwan showed that patients with depression correlate with 1.8-fold increased risk of gastrointestinal cancer $(95 \% \mathrm{CI}=1.03,3.14)$, but HCC is not mentioned specifically [2]. In the present study, despite the fact that we controlled the comorbidities potentially associated with HCC, no association is detected between depression 
and $\mathrm{HCC}$ risk in older people $(\mathrm{OR}=0.81,95 \% \mathrm{CI}=0.59,1.11)$. Generally speaking, $70-90 \%$ of all HCC cases are significantly associated with cirrhosis or other chronic the liver diseases worldwide [8]. Depression is a mood disorder, not causing chronic inflammation of liver. Therefore, these two diseases should have their unique pathogenesis without potential linking. Since formal depression diagnostic procedures were not recorded in this database, more prospective studies are necessary to validate our findings.

\section{Conflict of Interests}

The authors disclose no conflict of interests.

\section{Authors' Contribution}

Shih-Wei Lai: contributed substantially to the conception of this paper conducted the study, interpreted the data, initiated the draft of the paper and critically revised the article. ChengLi Lin conducted the statistical analysis and critically revised it. Kuan-Fu Liao and Wen-Chi Chen conducted the study and critically revised the paper.

\section{Acknowledgment}

The authors would like to thank the National Health Research Institute in Taiwan for providing the insurance claims data. This study was supported in part by Taiwan Department of Health Clinical Trial and Research Center of Excellence (DOH 102-TD-B-111-004) and China Medical University Hospital (Grant no. 1MS1). The funding agency did not influence the study design, data collection and analysis, decision to publish, or preparation of the paper.

\section{References}

[1] A. L. Gross, J. J. Gallo, and W. W. Eaton, "Depression and cancer risk: 24 years of follow-up of the Baltimore epidemiologic catchment area sample," Cancer Causes and Control, vol. 21, no. 2, pp. 191-199, 2010.

[2] Y.-H. Chen and H.-C. Lin, "Increased risk of cancer subsequent to severe depression-a nationwide population-based study," Journal of Affective Disorders, vol. 131, no. 1-3, pp. 200-206, 2011.

[3] M. E. J. Oerlemans, M. van den Akker, A. G. Schuurman, E. Kellen, and F. Buntinx, "A meta-analysis on depression and subsequent cancer risk," Clinical Practice and Epidemiology in Mental Health, vol. 3, article 29, 2007.

[4] J.-A. Liang, L.-M. Sun, C.-H. Muo, F.-C. Sung, S.-N. Chang, and C.-H. Kao, "The analysis of depression and subsequent cancer risk in Taiwan," Cancer Epidemiology Biomarkers \& Prevention, vol. 20, no. 3, pp. 473-475, 2011.

[5] S.-W. Lai, K.-F. Liao, C.-C. Liao, C.-H. Muo, C.-S. Liu, and F.C. Sung, "Polypharmacy correlates with increased risk for hip fracture in the elderly: a population-based study," Medicine, vol. 89, no. 5, pp. 295-299, 2010.

[6] S.-W. Lai, L.-T. Su, C.-H. Lin, C.-H. Tsai, F.-C. Sung, and D. P. H. Hsieh, "Polypharmacy increases the risk of Parkinson's disease in older people in Taiwan: a population-based study," Psychogeriatrics, vol. 11, no. 3, pp. 150-156, 2011.
[7] S.-W. Lai, P.-C. Chen, K.-F. Liao, C.-H. Muo, C.-C. Lin, and F.C. Sung, "Risk of hepatocellular carcinoma in diabetic patients and risk reduction associated with anti-diabetic therapy: a population-based cohort study," The American Journal of Gastroenterology, vol. 107, no. 1, pp. 46-52, 2012.

[8] D. A. Herbst and K. R. Reddy, "Risk factors for hepatocellular carcinoma," Clinical Liver Disease, vol. 1, pp. 180-182, 2012. 


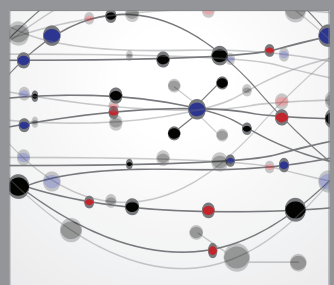

The Scientific World Journal
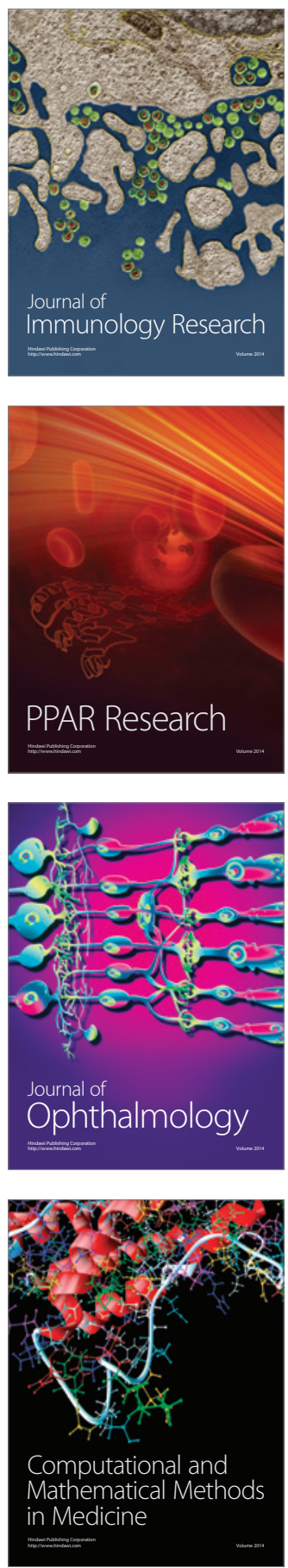

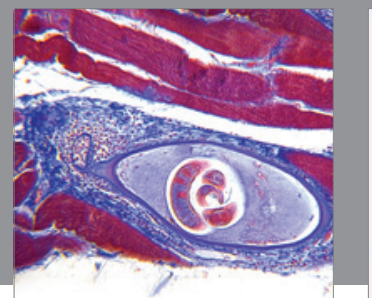

Gastroenterology

Research and Practice
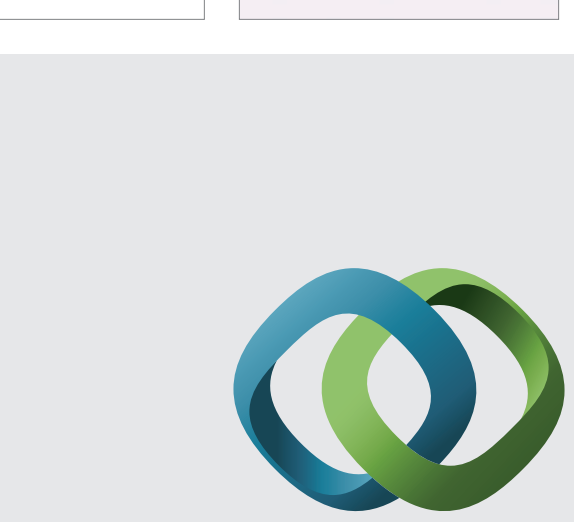

\section{Hindawi}

Submit your manuscripts at

http://www.hindawi.com
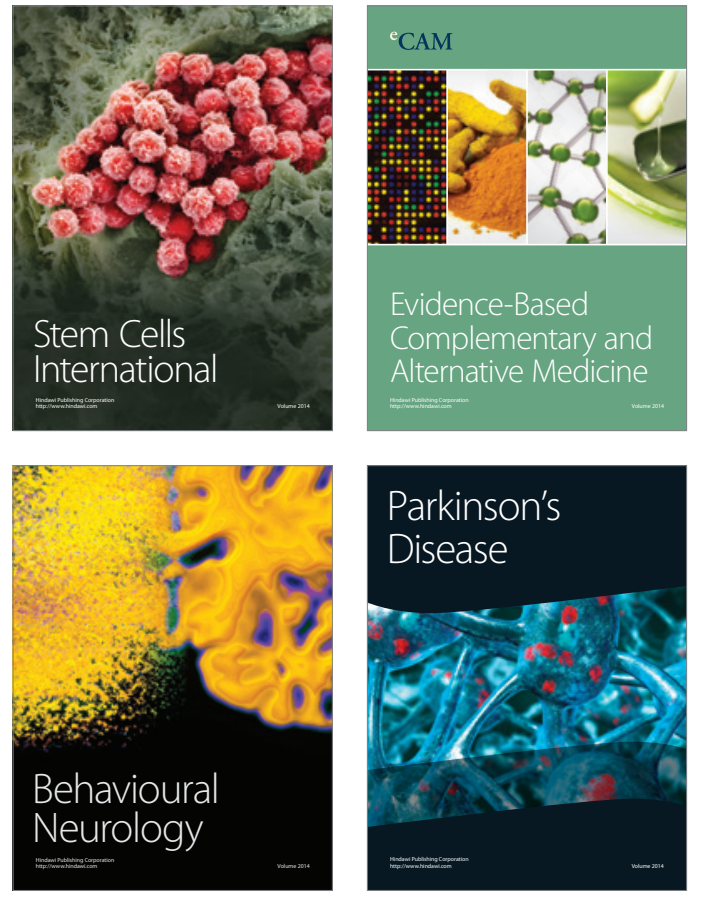
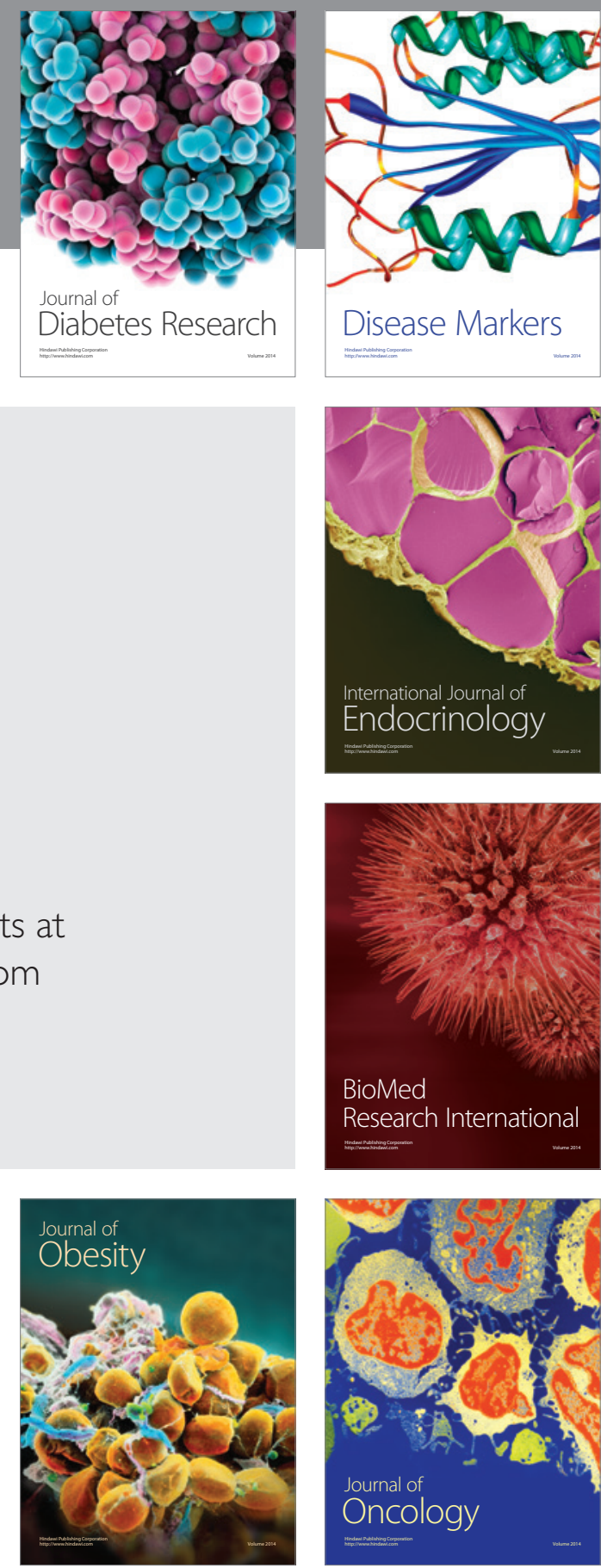

Disease Markers
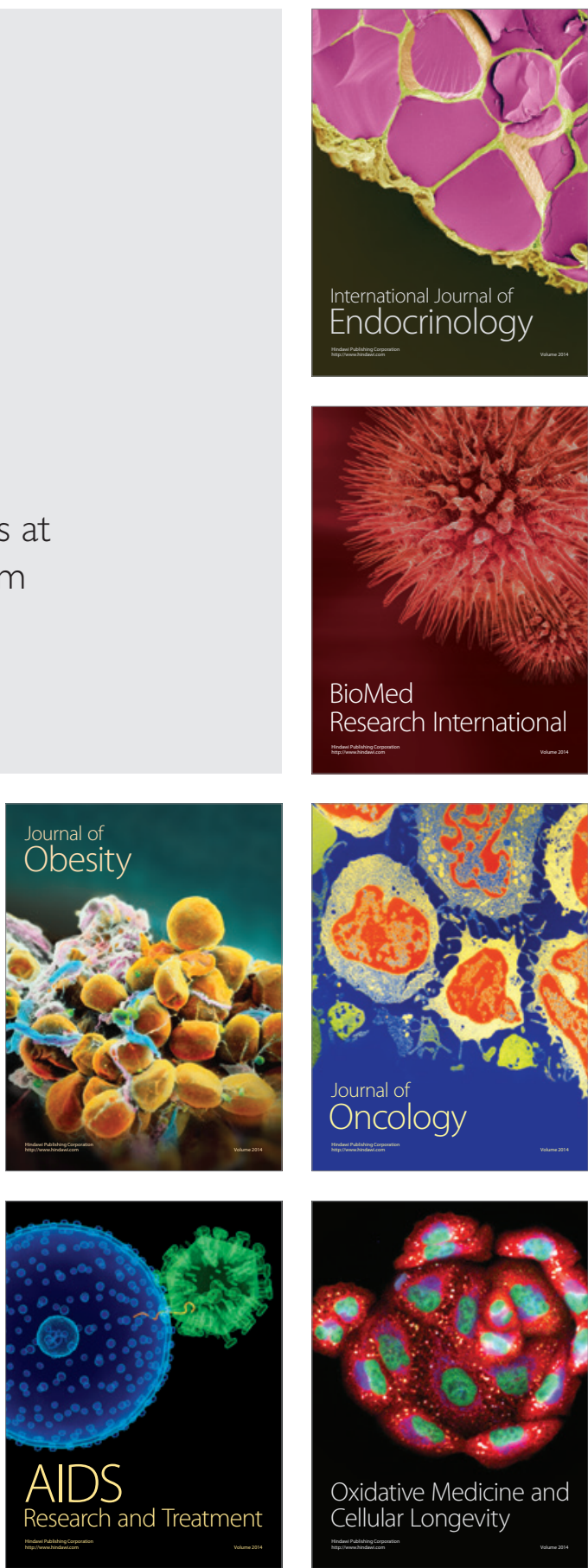